\title{
Analogy between dielectric relaxation and dielectric mixtures: Application of the spectral density representation
}

\author{
Enis Tuncer \\ E-mail: enis.tuncer@physics.org
}

\begin{abstract}
The spectral representation is an effecient tool to explore electrical properties of material mixtures. It separates the contributions of geometrical topology and intrinsic properties of the constituents in the system. The aim of this paper is to derive an expression for the spectral density representation, which favors dielectric relaxation phenomenon. This unfamiliar form is distinct in a way that the existing dielectric relaxation models and data analysis tools can be employed for extracting the spectral density function of a given system.
\end{abstract}

Electrical properties of material mixtures have attracted researchers in academia and industry to seek a relation between overall composite properties and intrinsic properties of the constituents and their spatial arrangement inside the mixture [1]. Bergman 2] has proposed a mathematical way for representing the effective dielectric permittivity $\varepsilon_{\mathrm{e}}$ of a binary mixture as a function of permittivities of its constituents, $\varepsilon_{\mathrm{m}}$ and $\varepsilon_{\mathrm{i}}$, and an integral equation, which includes the geometrical contributions. This theory is called the spectral density representation. Milton [3] gave the corrections, whereas, Golden and Papanicolaou 4] presented the rigorous derivation for the spectral representation. The permittivity $\varepsilon_{\mathrm{e}}$ of a mixture is expressed as follows in the spectral representation [5]

$$
\begin{aligned}
\varepsilon_{\mathrm{e}}= & \varepsilon_{\mathrm{m}}\left\{1+q A\left(\varepsilon_{\mathrm{i}} \varepsilon_{\mathrm{m}}{ }^{-1}-1\right)+\right. \\
& \left.\int_{0^{+}}^{1} q \mathrm{G}(x)\left[\left(\varepsilon_{\mathrm{i}} \varepsilon_{\mathrm{m}}{ }^{-1}-1\right)^{-1}+x\right]^{-1} \mathrm{~d} x\right\}
\end{aligned}
$$

where $q$ and $x$ are the concentration of inclusions and spectral parameter, respectively, and $\mathrm{G}(x)$ is the spectral density function. The constant $A$ is related to the percolation strength, which includes the contribution of $\mathrm{G}(0)$. Eq. (11) can be expanded by substituting $\varepsilon_{i}-\varepsilon_{j} \equiv \Delta_{i j}$, then Eq. (11) becomes,

$$
\begin{aligned}
\Delta_{\mathrm{em}} \varepsilon_{\mathrm{m}}{ }^{-1}= & q A \Delta_{\mathrm{im}} \varepsilon_{\mathrm{m}}{ }^{-1}+ \\
& \int_{0^{+}}^{1} q \mathrm{G}(x) \Delta_{\mathrm{im}}\left(\varepsilon_{\mathrm{m}}+\Delta_{\mathrm{im}} x\right)^{-1} \mathrm{~d} x
\end{aligned}
$$

Now, multiplying both sides with $\varepsilon_{\mathrm{m}}$ and letting $\Delta_{\mathrm{em}} / \Delta_{\mathrm{im}} \equiv \xi$ and $q A \equiv=\xi_{s}$, we obtain

$$
\xi=\xi_{s}+q \int_{0^{+}}^{1} \mathrm{G}(x)\left(1+\varepsilon_{\mathrm{m}}^{-1} \Delta_{\mathrm{im}} x\right)^{-1} \mathrm{~d} x
$$


Here, we call $\xi$ the 'scaled permittivity' and $\xi_{s}$ the percolation strength as defined earlier. The mathematical properties of $\mathrm{G}, q$ and $\xi_{s}$ are expressed in the literature [5]; Here $\xi_{s}+q \int_{0^{+}}^{1} \mathrm{G}(x) \mathrm{d} x=1$ and $\int_{0^{+}}^{1} x \mathrm{G}(x) \mathrm{d} x=(1-q) d^{-1}$, where $d$ is the dimensionality of the system. In view of Eq. (3) , the dielectric relaxation expression for a process with single relaxation time $\tau$ is expressed as $[\underline{6}, 7]$

$$
\varepsilon(\imath \omega)=\varepsilon_{\infty}+\Delta \varepsilon(1+\imath \omega \tau)^{-1}
$$

where $\varepsilon_{\infty}, \Delta \varepsilon$ and $\omega$ are the permittivity at optical frequencies $(\omega \rightarrow \infty)$, dielectric strength and angular frequency respectively. If there exist a continuous relaxation with a distribution, Eq. (41) becomes

$$
\varepsilon(\imath \omega)=\varepsilon_{\infty}+\Delta \varepsilon \int_{0}^{\infty} \mathrm{G}(\tau)(1+\imath \omega \tau)^{-1} \mathrm{~d} \tau .
$$

where $\mathrm{G}(\tau)$ is the distribution function of the relaxation times, and the static dielectric permittivity $\varepsilon_{s}$ is defined as $\varepsilon_{\infty}+\Delta \varepsilon \int_{0}^{\infty} \mathrm{G}(\tau) \mathrm{d} \tau=\varepsilon_{s}$. Observe the similarities between Eqs. (31) and (51). Although the resemblence between two equations looks superficial, when Claussius-Mossotti [ 6, 8] expression is taken into consideration, then the frequency dependent properties of dielectrics can infact be written as a dynamic response of dipole units embedded in a background medium $\varepsilon_{\mathrm{m}}$, concluding a dielectric mixture approach with inclusions as dipole units. Consequenctly, interfacial polarization observed in dielectric mixtures can be used to understand the structure-property relationship in physics of dielectrics.

There are couple of expressions that are extensively used in the dielectric data analysis, e.g. Havriliak-Negami [9], Davidson-Cole [10], Cole-Cole [1], which have known distribution functions $\mathrm{G}(\tau)$ [7]. In addition, there exists a vast literature on dielectric data analysis [7, 12]. The total polarizability of a material is given as $\varepsilon(\omega \rightarrow 0)$, therefore, $\varepsilon_{\infty}+\Delta \varepsilon=\varepsilon_{s}$, which is actually similar to the definitions and properties of $\mathrm{G}(x)$ and $\xi_{s}$, which can be obtained by converting polarizations to fractional polarizations, which results by dividing both sides by $\varepsilon_{s}$. As a consequence, we can complete the derivation and the hypothesis about the relation between the dielectric relaxation and two-component dielectric mixtures as the relationship between various parameters in both representations, $\varepsilon_{\infty} \varepsilon_{s}^{-1} \Leftrightarrow \xi_{s}, \Delta \varepsilon \varepsilon_{s}^{-1} \Leftrightarrow q$ and $\mathrm{G}(x) \Leftrightarrow \mathrm{G}(\tau)$ and $\omega \Leftrightarrow \varpi \equiv \varepsilon_{\mathrm{m}}^{-1} \Delta_{\mathrm{im}}$, where $\varpi$ is the scaled frequency of the mixture system.

Due to the similarities between Eqs. (3) and (5), and the commutability of various parameters, one can express a general 'scaled' dielectric mixture formula as in the dielectric dispersions, i.e. the Havriliak-Negami expression [9],

$$
\xi(\varpi)=\xi_{s}+q\left[1+(\varpi x)^{\alpha}\right]^{-\beta}
$$

As a result the dielectric permittivity $\varepsilon_{\mathrm{e}}$ of the mixture then becomes

$$
\varepsilon_{\mathrm{e}}=\varepsilon_{\mathrm{m}}+\Delta_{\mathrm{im}}\left\{\xi_{s}+q\left[1+(\varpi x)^{\alpha}\right]^{-\beta}\right\} .
$$

Yet the scaled permittivity notation can somewhat be used to calculate the timedomain dielectric mixture relations by using an inverse transform as in the case of dielectric relation theory, i.e. the relationship between the response function and the 
dielectric susceptibility. As note, one more resemblance between the two representations expressed by the Havriliak-Negami expression is that it yields the Maxwell Garnett equation [13], which formulates the dielectric permittivity of a mixture with spherical inclusions $(d=3)$, while in the dielectric relaxation form it results in the simple Debye relaxation in Eq. (4), when $\alpha=\beta=1, \xi_{s} \approx 0$ and $x=(1-q) d^{-1}$. Similarly for Claussius-Mossotti expression, $\alpha=\beta=1$, and the spectral parameter localizes at $3^{-1}$; and $q$ becomes the number density of dipoles.

In this short paper, it is despised that the dielectric relaxation phenomenon and expressions developed thereof for analyzing dielectric dispersions could be employed to investigate the topological description or spectral density functions of two-component composites. Once the data are transformed to the scaled permittivity notation as shown in Eqs. (3) or (6), the existing dielectric relaxation data analysis tools infact can readily be applied, there exist many dispersion expressions in the literature. Finally, it can be inferred that even pure one-component materials can also be expressed in terms of scaled permittivity notation, wherein, the smallest parts of the material are embedded in vacuum $\varepsilon_{\mathrm{m}}=1$. In such case the spectral density function would be indicative of the structure of the material and its parts, which in turn can be valuable to calculate the local fields [8] and interaction energies [14].

I thank Mr Rajeev Singh for fruitful discussion and for valuable comments while preparing this paper.

\section{References}

[1] R. Landauer, in Electrical Transport and Optical properties of Inhomogeneous Media, edited by J. C. Garland and D. B. Tanner (American Institute of Physics, New York, 1978), vol. 40 of AIP Conference Proceedings, pp. 2-43; L. V. Keldysh, D. A. Kirzhnitz, and A. A. Maradudin, eds., The Dielectric Function of Condensed Systems (Elsevier Science Publisher B.V., Amsterdam, 1989); A. Priou, ed., Progress in Electromagnetics Research, Dielectric Properties of Heterogeneous Materials (Elsevier, New York, 1992); D. J. Bergman and D. Stroud, Solid State Physics 46, 147 (1992); A. Sihvola, Electromagnetic mixing formulas and applications, vol. 47 of IEE Electromagnetic Waves Series (The Institute of Electrical Engineers, London, 1999).

[2] D. J. Bergman, Phys. Rep. 43(9), 377 (1978); D. J. Bergman, Phys. Rev. Let. 44(19), 1285 (1980); D. J. Bergman, Ann Phys 138, 78 (1982).

[3] G. W. Milton, J Appl Phys 52, 5286 (1981); G. W. Milton, J Appl Phys 52(8), 5294 (1981).

[4] K. Golden and G. Papanicolaou, J Stat Phys 40(4/5), 655 (1985).

[5] K. Ghosh and R. Fuchs, Phys Rev B 38(8), 5222 (1988); A. V. Goncharenko, V. Z. 
Lozovski, and E. F. Venger, Opt Comm 174, 19 (2000); A. V. Goncharenko, Phys Rev E 68, 041108 (2003).

[6] P. Debye, Polar Molecules (Dover Publications, New York, 1945).

[7] E. Tuncer and S. M. Gubański, IEEE Trans Diel El Insul 8, 310 (2001).

[8] L. Onsager, J Am Chem Soc 58, 1486 (1936); H. Fröhlich, Theory of Dielectrics; Dielectric constant and dielectric loss (Oxford Science Publications, Oxford, 1958), 2nd ed; U. Fano, Phys Rev 118, 451 (1960); S. E. Schnatterly and C. Tarrio, Revi Mod Phys 64, 619 (1992); B. K. P. Scaife, Principles of Dielectrics (Oxford Science Publications, 1998).

[9] S. Havriliak and S. Negami, J Polym Sci: Part C 14, 99 (1966).

[10] D. W. Davidson and R. H. Cole, J Chem Phys 19, 1484 (1951).

[11] K. S. Cole and R. H. Cole, J Chem Phys 9, 341 (1941).

[12] A. K. Jonscher, Dielectric Relaxation in Solids (London: Chelsea Dielectric, London, 1983); J. R. Macdonald, ed., Impedance Spectroscopy (John Wiley \& Sons, New York, 1987); J. R. Macdonald, J Comp Phys 157, 280 (2000); J. R. Macdonald, J Non-Cryst Solids 212, 95 (1997); J. R. Macdonald and L. D. Potter Jr., Solid State Ionics 24(1), 61 (1987).

[13] J. C. M. Garnett, Phil Trans Royal Soc London A 203, 385 (1904); O. Levy and D. Stroud, Phys Rev B 56(13), 8035 (1997).

[14] R. G. Barrera and R. Fuchs, Phys Rev B 52, 3256 (1995); J. Monecke, Phys Rev B 55, 7515 (1997); R. O. Kuzian, R. Hayn, and A. F. Barabanov, Physical Review B 68, 195106 (2003); M. I. Stockman, D. J. Bergman, and T. Kobayashi, Physical Review B 69, 054202 (2004). 